\title{
Cryoprobe with T-tip for retinal surgery
}

\author{
M. G. LYALL \\ From the Regional Eye Centre, Oldchurch Hospital, Romford, Essex
}

A cryoprobe with a T-tip designed to facilitate cryotherapy in retinal surgery is described in this paper.

The T-tip cryoprobe (Figs I and 2) is modelled on the thimble indentor of Schepens (1950). In

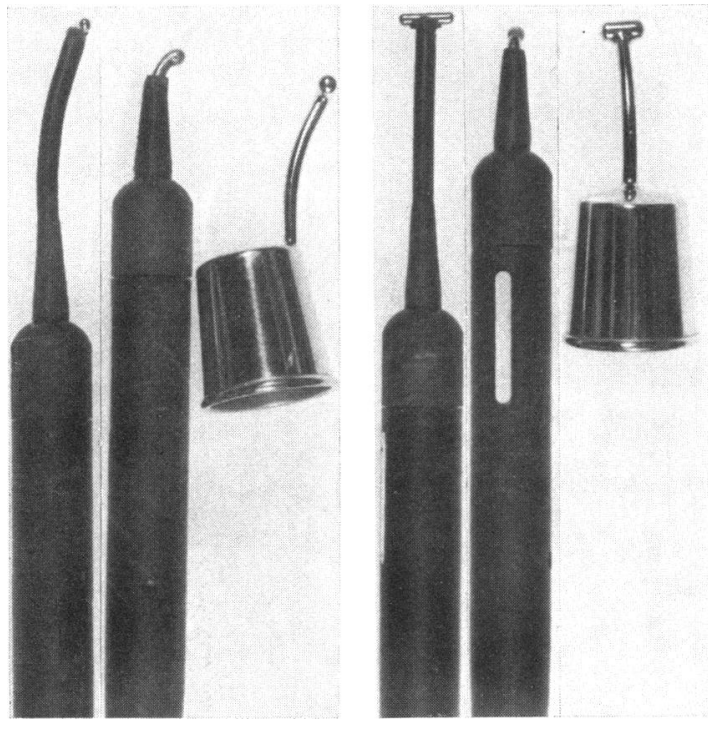

FIG. I and FIG. 2 T-tip cryoprobe, conventional cryoprobe, and thimble indentor

my experience the conventional cryoprobe when used as an indentor in monitored cryopexy operations may show up retinal holes and tears less well than the thimble indentor. Also it may be difficult to manoeuvre the conventional probe, so that a large tear is completely surrounded by treated areas. Furthermore, as Chignell, Revie, and Clemett (197I) indicate, when there is a large area to be treated refreezing may be carried out inadvertently with danger of pigment fall-out and macular damage. Lastly, when a large area has to be treated -for example, in peripheral retinal dialysis-it takes a long time to give the large number of applications necessary. The T-tip probe treats a larger area with each application and so reduces

Address for reprints: M. G. Lyall, FRCS, Oldchurch Hospital, Regional Eye Centre, Roniford, Essex the number of applications needed. Time-saving techniques are advantageous, since operative concentration and skill tend to fall off after two hours.

\section{Method of use}

Obviously, the T-probe indents as the thimble indentor. Most tears are dealt with readily by three applications. The first is given so that the freeze reaches the posterior rounded margin of the tear, the second is given across the tear, and the third touching the anterior angles of the tear (Fig. 3). After the first application it is a simple matter to bring the probe forwards for the next freeze and then the third. Areas of lattice or lattice with holes are readily treated, since they are usually disposed in a circumferential fashion and one or two applications in any meridian are usually enough. Similarly, dialyses can be conveniently treated.

Monitoring shows that the freeze appears first in one area of the indent and then spreads to the remainder. As with the conventional probe, the freeze is delayed in transconjunctival cryotherapy when an area of choroid deep to a muscle is being treated. Satisfactory pigmentation of Chignell's Grade II type (Chignell and others, I 97I) is produced with appropriate application. I have used the probe in over 20 cases, producing with it a

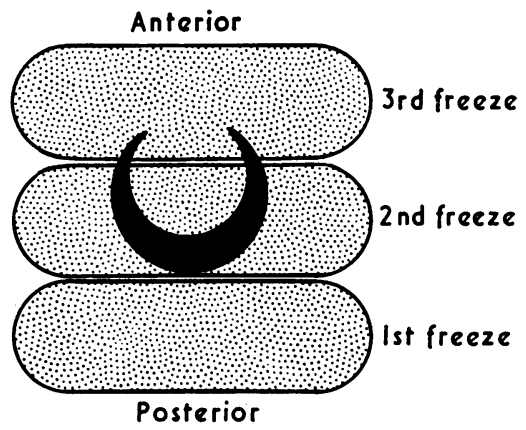

FIG. 3 Freeze pattern in treatment of common horseshoe tear

pigment macular fall-out in one case in which there were three large horseshoe tears and macular detachment.

The T-tip cryoprobe attaches to Amoils cryo unit. It is available from Keelers, London. A similar model for use with the MCrooo cryosurgical system is available from KeyMed, Southend-on-Sea.

\section{References}

Chignell, A. H., Revie, I. H. S., and ClemetT, R. S. (I97I) Trans. ophthal. Soc. U.K., 91, 635 schepens, c. L. (1950) XVI Concil. Ophthal., 2, 1384 (quoted in Eye Surgery, by H. B. Stallard p. 671) 